\title{
A Methodology for Rapid Prototyping Peak-Constrained Least-Squares Bit-Serial Finite Impulse Response Filters in FPGAs
}

\author{
Alex Carreira \\ Department of Electrical and Computer Engineering, University of Calgary, 2500 University Drive N.W., \\ Calgary, Alberta, Canada T2N 1N4 \\ Email:aycarrei@shaw.ca \\ Trevor W. Fox \\ Department of Electrical and Computer Engineering, University of Calgary, 2500 University Drive N.W., \\ Calgary, Alberta, Canada T2N 1N4 \\ Email:fox@enel.ucalgary.ca \\ Laurence E. Turner \\ Department of Electrical and Computer Engineering, University of Calgary, 2500 University Drive N.W., \\ Calgary, Alberta, Canada T2N 1N4 \\ Email: turner@enel.ucalgary.ca
}

Received 28 February 2002 and in revised form 17 October 2002

\begin{abstract}
Area-efficient peak-constrained least-squares (PCLS) bit-serial finite impulse response (FIR) filter implementations can be rapidly prototyped in field programmable gate arrays (FPGA) with the methodology presented in this paper. Faster generation of the FPGA configuration bitstream is possible with a new application-specific mapping and placement method that uses JBits to avoid conventional general-purpose mapping and placement tools. JBits is a set of Java classes that provide an interface into the Xilinx Virtex FPGA configuration bitstream, allowing the user to generate new configuration bitstreams. PCLS coefficient generation allows passband-to-stopband energy ratio (PSR) performance to be traded for a reduction in the filter's hardware cost without altering the minimum stopband attenuation. Fixed-point coefficients that meet the frequency response and hardware cost specifications can be generated with the PCLS method. It is not possible to meet these specifications solely by the quantization of floating-point coefficients generated in other methods.
\end{abstract}

Keywords and phrases: placement, mapping, FIR filter, PCLS, bit serial, JBits.

\section{INTRODUCTION}

Finite duration impulse response (FIR) digital filters are critical components in a wide spectrum of digital signal processing (DSP) operations and systems. Examples include: decimation, radar, and image processing [1]. Rapid prototyping of FIR filters is important in reducing development time and costs. Previous research efforts have focused on implementation and system architecture $[2,3,4]$ with little or no attention paid to methods for rapid prototyping. Filter performance should not be sacrificed in a rapid prototyping methodology for FIR filters. A recent design that can be used to rapidly prototype FIR filters [5] uses a windowing technique that sacrifices the ability to precisely control the frequency response performance of the filter [1].
The FIR filter frequency response performance can be controlled by the method of peak-constrained least-squares (PCLS), which allows both the minimum stopband attenuation and the passband-to-stopband energy ratio (PSR) to be controlled [6]. A method for rapidly prototyping PCLS bit-serial FIR filters that is able to trade PSR performance for reduced hardware area in the FPGA without altering the minimum stopband attenuation is described in this paper. Fixed-point coefficients that meet the frequency response and hardware cost specifications can be generated with the PCLS method. It is not possible to meet frequency response and hardware specifications solely by quantizing floatingpoint coefficients generated by other methods (least-squares and Parks-McClellan [1]) to fixed-point coefficients. Previously presented PCLS methods $[6,7,8,9]$ have not been used for rapid prototyping of FIR filters. 
Reduction of the Field Programmable Gate Array (FPGA) hardware resources used to implement this FIR filter and increased hardware density is facilitated by an areaefficient bit-serial FIR filter architecture [10] at the expense of a lower sample rate. We have developed further area efficiency results from a bit-serial filter core library for JBits along with an application-specific mapping and placement strategy that is presented in the paper. Hardware density of the implementation is increased while avoiding the timeconsuming place and route processes required in conventional tools that synthesize FPGA configuration bitstreams.

The Java language is used in conjunction with the JBits application program interface (API) and JBits runtime parameterizable (RTP) cores [11] to rapidly prototype a PCLS bit-serial FIR filter. JBits is a set of Java classes that provide an interface into the Xilinx Virtex FPGA configuration bitstream, allowing the user to generate configuration bitstreams [12]. Most of the resources of the FPGA, for instance, the configurable logic blocks (CLBs), routing switches and multiplexers, and input-output blocks (IOBs) can be accessed and configured by using JBits method calls. JBits method calls perform modifications to the FPGA at a very low level [13] and consequently developing a large application with such calls can be more difficult than using a highlevel hardware description language (HDL).

A core is a predesigned logic module that removes the need to implement an entire design in low-level detail [11]. While low-level elements can also be represented by a core, for instance an AND gate, the JBits RTP core specification provides a means for the design to be completed at a level of abstraction similar to that of traditional HDLs [13]. The difference between a JBits RTP core and cores used in traditional structural HDLs is that each JBits core must be physically placed and interconnected within the FPGA during implementation [13]. JBits provides means to place the cores relative to other cores or by explicitly defining the coordinates of the core within the FPGA.

Traditional FPGA-based designs can be hierarchically built from a library of static cores that elaborate to a netlist [5] of fine grained subcomponents that can be implemented in an FPGA-based design using a time-consuming place and route process. Because the static cores elaborate to a netlist, there is no requirement that the subcomponents that are used to create the static core be placed in advance. The core exists only as a definition of subcomponents within the FPGA's fabric. In JBits, RTP cores are used instead of static cores. RTP cores differ significantly because they elaborate into an FPGA configuration bitstream instead of a netlist [5]. The subcomponents of an RTP core must have a predefined physical placement because they are not used with traditional place and route tools. In an FPGA, RTP cores have a fixed shape known as a bounding box that may dimensionally vary, based on the core's parameters; for instance, a register core may have a fixed-height bounding box that grows horizontally with the number of bits specified in the register's width parameter. The often irregular and dissimilar sizes of different cores that may be used in a JBits-based hierarchical design lead to a placement problem that may be complex and time consuming or impossible to solve if a high level of hardware density is desired.

The placement director described in this paper extends the ability to explicitly define coordinates of JBits RTP cores within the FPGA with methods that place cores in the FPGA in a folded fashion to maximize hardware density of a bitserial FIR filter core implemented in JBits. This technique requires that all the subcores that are placed with the placement director in the FPGA have an identical width dimension when implemented in the FPGA fabric.

Faster generation of the FPGA configuration bitstream obtained by avoiding conventional general-purpose mapping and placement tools is possible for a bit-serial FIR filter core by using the application-specific mapping and placement method for JBits. This is further described in Section 4. JBits does not directly support bit-serial system implementations, necessitating the creation of a library of pipelined bitserial arithmetic operator cores. Each core in the pipelined bit-serial arithmetic operator library is precoded in the Java programming language as an RTP core. Every core in the library of bit-serial RTP cores processes a width dimension of one slice when implemented in the FPGA fabric. This core library can be used to construct a PCLS bit-serial FIR filter, which is further explained along with the system architecture in Section 2. The design of bit-serial PCLS filters is discussed in Section 3. The process of generating hardware to implement a set of filter coefficients is described in Section 4 . The PSR and hardware cost trade-off are discussed in Section 5 and the layout of a PCLS FIR filter is presented in Section 6.

\section{ARCHITECTURE}

High sample-rate FIR filters are not required in all FPGAbased DSP systems. It is possible to use filter architectures that trade sample-rate performance for additional area efficiency to implement filters [14]. Bit-serial architectures can be used to construct the FIR filters in these systems with the following benefits:

(i) reduced hardware size because less hardware and interconnect area are needed for bit-serial implementations;

(ii) simplified subcomponent placement. Bit-serial components are small and similarly shaped, resulting in simplified alignment of the components when placing a design;

(iii) increased hardware utilization and hardware density. Small size and similar shape means that space is not wasted due to gaps or irregular fit between adjacent bit-serial library components in a placement.

Hardware area savings or area efficiency in the bit-serial architecture comes at the expense of reduced sample rate compared to a bit-parallel design.

\subsection{Filter architecture}

A rearrangement of the direct form FIR filter architecture into the transposed FIR filter architecture [10] is beneficial 
TABLE 1: Summary of data for bit-serial component library.

\begin{tabular}{llccl}
\hline Component & Width & Height & Latency (cycles) & Functionality \\
\hline FD (one-bit register) & 1 slice & $1 \mathrm{LE}$ & 1 & Positive coefficient MSB in a coefficient multiplier. \\
FDIR slice & 1 slice & $1 \mathrm{LE}$ & 1 & A coefficient zero bit in a coefficient multiplier. \\
Carry-save adder slice & 1 slice & $2 \mathrm{LEs}$ & 1 & A coefficient one bit in a coefficient multiplier. Carry-save adder from [2]. \\
Tap adder slice & 1 slice & $2 \mathrm{LEs}$ & 1 & Adder for delay and coefficient multiplier outputs. Carry-save adder from [2]. \\
TDS & 1 slice & $2 \mathrm{LEs}$ & $1-32$ & Unit sample delay. Delay from [2]. \\
Two's complement slice & 1 slice & $2 \mathrm{LEs}$ & 1 & Negative MSB bit in a coefficient multiplier. Two's complement from [2]. \\
\hline
\end{tabular}

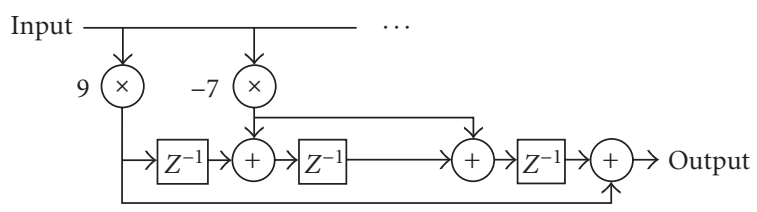

FIGURE 1: Modified transversal filter architecture implementing coefficient set $\{9,-7,-7,9\}$. Coefficient multipliers are shared for duplicated coefficients in the coefficient set.

to construction of a bit-serial FIR filter by reducing required hardware and control signals.

The latency of a bit-serial component is the time delay for output data to be generated from the time that data is input to the component. A benefit of the transposed architecture is the absence of the direct form architecture adder tree, which requires additional control signals for each adder tree layer and exhibits increased latency.

The hardware resources required to implement the filter can be further reduced if duplicated coefficients are present in the coefficient set. The sharing of multipliers for duplicate coefficients in the transposed FIR filter architecture leads to the use of a single multiplier for each unique coefficient. The output of this multiplier then connects to the appropriate tap adders of the filter. A transposed filter architecture showing two coefficient multipliers for a filter with coefficient set $\{9,-7,-7,9\}$ is given in Figure 1.

\subsection{Bit-serial component library}

In order to hierarchically construct an FIR filter in an FPGA, an architecture-specific bit-serial core library is required. The advantage of bit-serial library cores for rapid prototyping of an FPGA-based DSP system is the small and similar area of the components and shorter interconnections between components.

JBits does not directly support bit-serial system implementations, necessitating the creation of a library of pipelined bit-serial arithmetic operator cores. Each core in the pipelined bit-serial arithmetic operator library is precoded in the Java programming language as an RTP core, however the application described in this paper uses the RTP cores as parameterizable static cores. An example of parameterization would be a register core that uses a parameter to define its width - thereby creating a register of varying width depending on the parameter. Traditional FPGA design tools

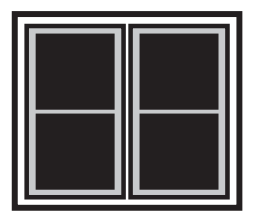

CLB

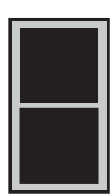

Slice

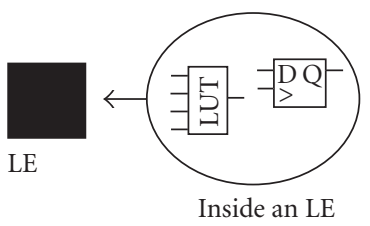

Figure 2: Relationship between CLBs, slices, and LEs. provide a library of predefined cores, for example, flip-flops, AND gates, adders, inverters, and many more cores that are not parameterized [11]. RTP cores are an extension of the traditional static core model that can be created at runtime and support runtime parameterization of designs [11]. That is, they are not instantiated during runtime but during the creation of the FPGA configuration bitstream.

The components of the pipelined bit-serial library are adder (carry-save adder), two's complement, and delay as described in [2]. For simplicity, a serial-by-parallel multiplier architecture [2] with signed two's complement coefficient coding was chosen over a multiplier with canonic signed digit (CSD) coding [10]. Constant coefficient CSD multiplier architectures can be less regular and therefore more difficult to construct than the method described in [2].

An understanding of the Virtex FPGA architecture is important to contrast the size of the bit-serial library components presented in Table 1. The Virtex FPGA is comprised of CLBs and IOBs. The Virtex FPGA is a large block of CLBs surrounded by a ring of IOBs. IOBs are not used in the bitserial component library and are not discussed herein.

Each CLB fits in a CLB column. Within a single CLB lies two slices; within each slice lie two logic elements (LEs). A depiction of the relationship between CLBs, slices, and LEs appears in Figure 2.

Within each LE are a four-input lookup table, a flip-flop, and additional logic to assist with specific common applications (e.g., fast-carry logic and 16-bit shift register lookup tables SRL16s). Using the lookup table, flip-flops, and additional LEs, it is possible to construct every bit-serial library component. More information on the Virtex architecture can be found in [15].

The pipelined bit-serial library we have built is similar to the library described in [2], but has been extended to simplify the construction of serial-by-parallel multipliers as 
described in [2] for constant coefficients. The construction has been simplified by providing additional library components for the negative most significant bit (MSB), positive $\mathrm{MSB}$, zero, and one-bit values in coefficients. For instance, there is a core exclusively for a one bit in a coefficient and another core for a zero bit. The cores also reduce area for zero bits in coefficients, because a zero bit can be implemented as a delay with inverted synchronous reset which is smaller than using a carry-save adder in FPGA hardware. The resulting pipelined bit-serial component library consists of the RTP cores shown in Table 1. Table 1 also shows the size of the cores in a Virtex FPGA, the latency of each core, and a brief description of the functionality of each core and which library part it implements in [2].

The carry-save adder slice is used to create a one-valued coefficient bit in the multiplier and differs from a tap adder slice in name to distinguish between carry-save adders used in coefficient multipliers and carry-save adders used to add up tap outputs in the delay line of Figure 1. An FDIR slice is a one-bit register with inverted synchronous reset that can be used to create zero-valued coefficient bits in the multiplier.

It is interesting to contrast the dimensions of the cores in Table 1 with the dimensions of a mid-range Virtex part. For example, an XCV 300 part is 96 slices wide by 64 LEs high. This could fit 3072 of the largest cores in the bit-serial library summarized in Table 1.

\subsection{Implementing a constant coefficient serial-by-parallel multiplier}

A constant coefficient serial-by-parallel coefficient multiplier architecture can be implemented from the bit-serial component library presented in Table 1 . To build a serial-by-parallel coefficient multiplier, a finite precision coefficient must be converted to a binary number with a minimum number of bits. For example, in a bit-serial system with eight-bit system word length (SWL), coefficient -5 would be converted to 1011 instead of 11111011 because the additional leading bits are not required for implementation. In the same bitserial system, coefficient 11 would be converted to 1011 instead of 000001011.

The binary number obtained from converting the finite precision coefficient is used to choose the cores to implement the multiplier. Any bit position other than the MSB is assigned a carry-save adder slice core for a one-valued bit or an FDIR slice core for a zero-valued bit. The MSB bit position is different because it requires choosing a two's complement slice core for negative coefficient MSBs or a flip-flop (FD core) for positive coefficient MSBs.

In Figure 3, the finite precision coefficient 11 has been converted to the binary number 1011. Using the binary number 1011 to assign the cores in the multiplier implementation leads to an FD core followed by an FDIR slice core and two carry-save adder slice cores. These cores are placed adjacent to each other, one on top of the other as shown in Figure 3. Placement order of the subcores is important to shorten interconnect that connects the out pins to the data pins of the adjacent cores. The input is applied at the core that corresponds to the MSB, while the output is derived from the core

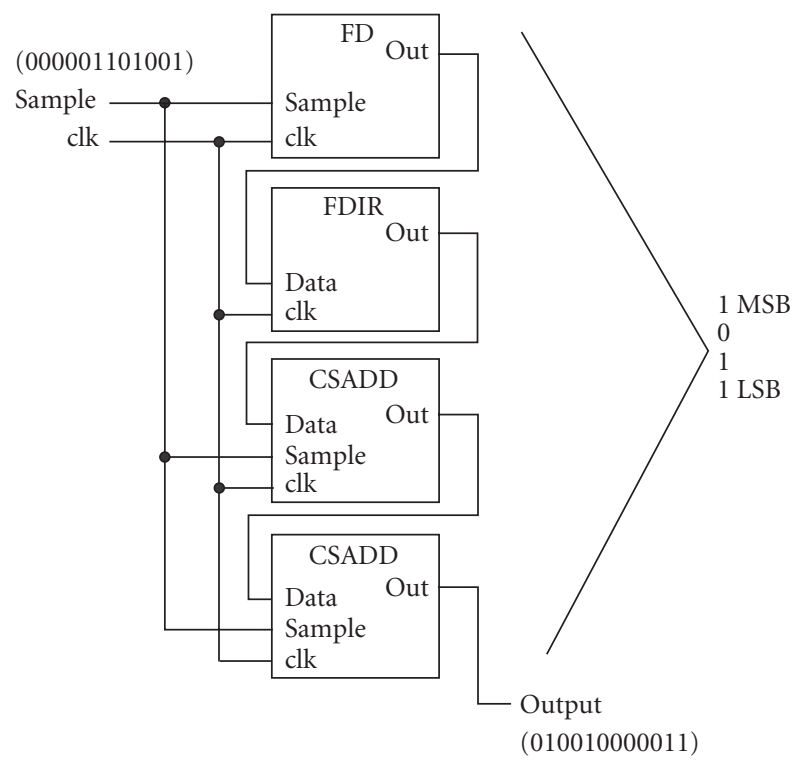

$$
\begin{aligned}
& \text { FD = Flip-flop } \\
& \text { CSADD = Carry-save adder slice } \\
& \text { FDIR = FDIR slice (flip-flop with inverted synchronous) }
\end{aligned}
$$

FIGURE 3: Serial-by-parallel constant coefficient multiplier for coefficient eleven, constructed from bit-serial component library. A control signal is not shown to simplify the diagram.

that corresponds to the binary number's LSB. The sample signal is an LSB first serial multiplicand, that is, multiplied by the coefficient multiplier to yield a serial product which appears 1 bit-time later at output. Further information on constructing serial-by-parallel multipliers can be found in [2].

\section{THE DESIGN OF BIT-SERIAL PEAK-CONSTRAINED LEAST SQUARES FIR FILTERS}

The method of PCLS can be used to generate finite precision coefficients that control the minimum stopband attenuation, PSR, and hardware cost [8, 9] of FIR filters. Quantization of floating-point coefficients for implementation in finite precision digital systems affects the filter frequency response performance. Finite precision coefficients generated by PCLS can be directly implemented without quantization ensuring correct frequency response performance. Least squares and minimax (equiripple) stopbands can be obtained using the PCLS methods described in $[6,7,8,9]$. Neither least squares nor minimax stopbands are effective at removing unwanted signals with wideband and narrowband components $[6,7]$. The method of PCLS can be used to design FIR filters with high PSR and minimum stopband attenuation values that are better suited to remove signals with wideband and narrowband components [6, 7]. Significant savings in hardware cost can be achieved at the expense of a slight reduction in PSR $[8,9]$.

The method of PCLS described in $[8,9]$ constrains an estimate of the hardware cost (the number of coefficient adders 
and subtractors) $[8,9]$. This design procedure has been extended to support the rapid design of bit-serial PCLS FIR filters using exact hardware cost, measured in Xilinx Virtex LEs. This new design procedure provides the ability to trade PSR performance for reduced hardware use in the filter core without altering the minimum stopband attenuation.

\subsection{Problem statement and formulation}

The design problem can be stated as follows: find an FIR transfer function that approximates a desired brick wall transfer function $H_{d}\left(e^{j 2 \pi f}\right)$ with $\delta_{p}$ maximum passband ripple and $\delta_{s}$ maximum stopband ripple, and using at most MaxLE number of LEs in the entire FIR implementation.

This problem can be formulated as a discrete PCLS optimization problem. Choose the discrete coefficients, $h$, to minimize the weighted squared error

$$
\varepsilon(h)=\int_{0}^{0.5} W\left(e^{j 2 \pi f}\right)|| H\left(e^{j 2 \pi f}\right)|-| H_{d}\left(e^{j 2 \pi f}\right)||^{2} d f
$$

subject to

$$
\begin{array}{ll}
|| H\left(e^{j 2 \pi f}\right)|-| H_{d}\left(e^{j 2 \pi f}\right)||-\delta_{p} \leq 0 & \text { for } f=\left[0, f_{p}\right], \\
|| H\left(e^{j 2 \pi f}\right)|-| H_{d}\left(e^{j 2 \pi f}\right)||-\delta_{s} \leq 0 & \text { for } f=\left[f_{s}, 0.5\right],
\end{array}
$$

$$
\operatorname{LE} \text { required }(h)-\operatorname{Max} \mathrm{LE} \leq 0,
$$

where $W\left(e^{j 2 \pi f}\right)$ is the squared error weighting function. The constants $f_{p}$ and $f_{s}$ are the passband and stopband cutoff frequencies, respectively. LE required $(h)$ is the total number of LEs required to implement the entire FIR filter. The discrete Lagrangian local search presented in $[8,9]$ can be used to solve this discrete PCLS optimization problem without modification. Once the coefficients are generated, they can be converted into hardware as discussed in the next section.

\section{CONVERTING COEFFICIENT VALUES INTO HARDWARE}

In this section, a new methodology for the construction of a bit-serial FIR digital filter using small, similar sized library components is presented. This method provides fast generation of the FPGA configuration bitstream with a new application-specific mapping and placement method that is similar to the linear layout of cells in a bit-serial VLSI chip design described in [10]. We have implemented this method in the JBits environment to avoid time-consuming generalpurpose mapping and placement tools commonly used to synthesize configuration bitstreams.

Finite precision coefficients generated using the local search method are converted into hardware in the bit-serial filter RTP core. This complex procedure can be divided into smaller subtasks. The subtasks are mapping, placement, and routing. Each subtask is described in more detail in Sections 4.1, 4.2, and 4.3.

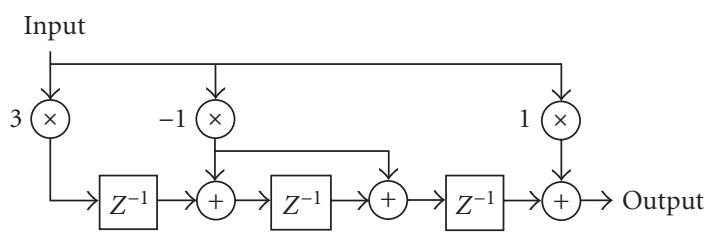

(a)

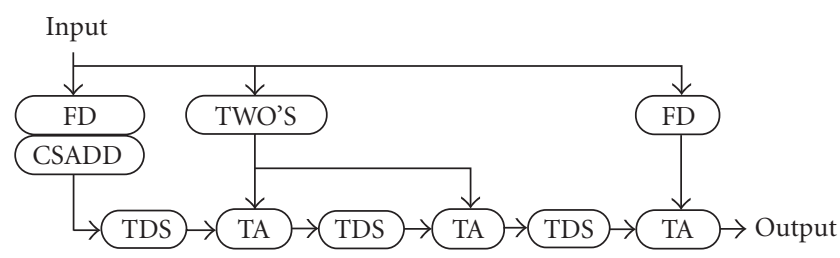

(b)

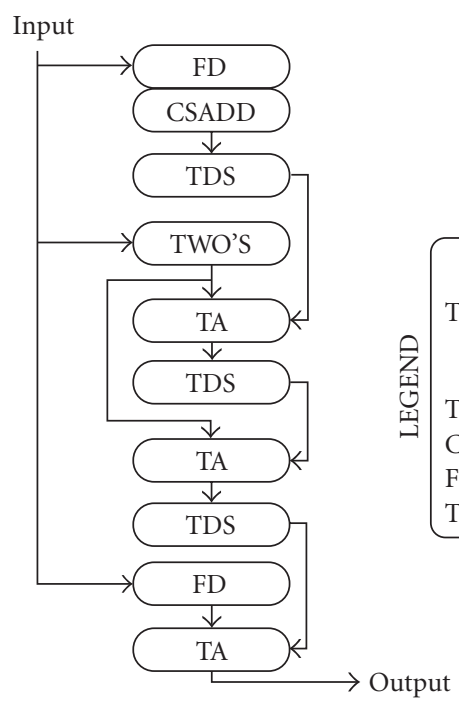

(c)

Figure 4: (a) Transposed FIR filter architecture for coefficient set $\{1,-1,-1,3\}$. (b) Cores substituted into the transposed FIR filter architecture to create constant coefficient serial-by-parallel multipliers, tap adders, and tap delays. (c) Transposed FIR filter architecture rearranged into a column of cores.

\subsection{Mapping: serial mapper}

The bit-serial filter core is the top-level core in a hierarchy of cores that implement a bit-serial FIR filter. The subcores within the bit-serial filter core are the bit-serial library components described in Table 1. The serial mapper is a data structure that maps the position of each subcore relative to the other subcores in the filter. Two one-dimensional lists (or serial maps) are contained in the data structure: a symbolic serial map that contains all the cores in the filter and a physical serial map that indicates which cores are assigned to each LE. Symbolic serial maps are composed of a column of cores. The physical serial map is a column of LEs that is used to determine FPGA hardware requirements for optimization equation (3) and placement of the cores in hardware. Figure 4 illustrates how the filter architecture of Figure 1 is 


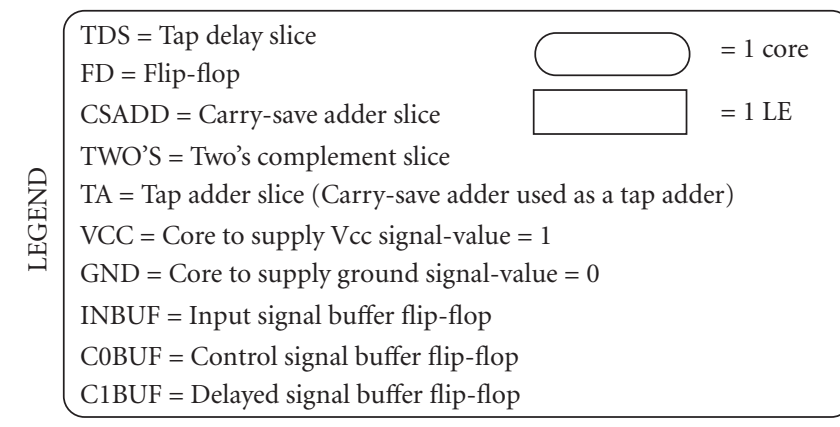

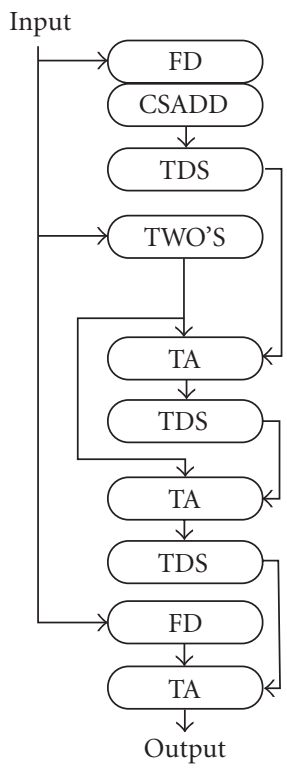

(a)

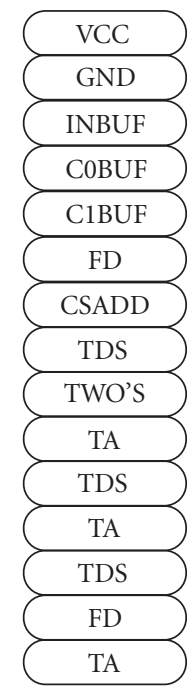

Symbolic serial map

(b)

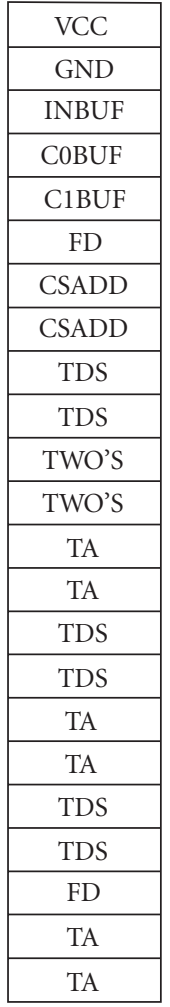

Physical serial map

(c)

Figure 5: (a) Transposed FIR filter architecture rearranged into a column of cores for coefficients $\{1,-1,-1,3\}$. (b) Symbolic serial map generated by the serial mapper for coefficient set $\{1,-1,-1,3\}$. The symbolic serial map corresponds to the transposed FIR filter architecture rearranged in (a). (c) Physical serial map generated by the serial mapper for coefficient set $\{1,-1,-1,3\}$, corresponding to the symbolic serial map in (b).

transformed into a column of cores for the coefficient set $\{1,-1,-1,3\}$.

In Figure $4 \mathrm{a}$, a transposed FIR filter is shown for the coefficient set $\{1,-1,-1,3\}$. Figure $4 \mathrm{~b}$ shows the result of substituting cores into the transposed FIR filter of Figure 4a. Note that constant coefficient multipliers of Figure $4 \mathrm{~b}$ are built from cores using the method shown in Figure 3. Figure 4c shows the rearrangement of Figure $4 \mathrm{~b}$ into a column of cores. Figure $4 \mathrm{c}$ retains signal arrows to show that the signal flow of Figure $4 \mathrm{~b}$ is unchanged in the structural transformation to a column of cores.

Figure 5, illustrates maps generated by the serial mapper from the coefficients $\{1,-1,-1,3\}$.

The symbolic serial map in Figure $5 \mathrm{~b}$ and the physical serial map in Figure 5c are discussed further in the next two sections.

\subsubsection{Symbolic serial map}

The symbolic serial map of Figure $5 \mathrm{~b}$ is constructed from the coefficient set $\{1,-1,-1,3\}$. The first five cores (starting from the top of Figure $5 b$ ) are used by the filter to create ground and Vcc nets and input buffers for the serial input and control signals. The next two cores are a coefficient multiplier corresponding to the coefficient 3 . The next core is a tap-delay slice (TDS) because a tap adder slice is not needed for the first coefficient in the architecture of Figure 1. After the TDS, one core is mapped to create a coefficient multiplier for the coefficient -1 . This core is followed by a tap adder slice and a TDS. Following the tap adder slice and TDS is another tap adder slice and another TDS because the coefficient multiplier for -1 is shared as shown in Figure 5 a. Further discussion of sharing coefficient multipliers appears in Section 4.1.4. The last two cores are used to create 


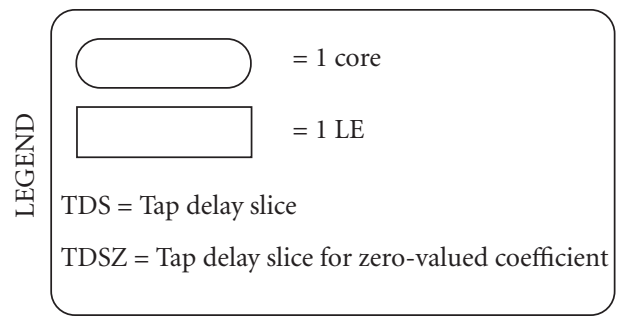

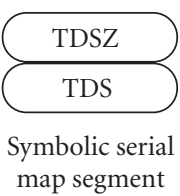

(a)

\begin{tabular}{|c|}
\hline TDSZ \\
\hline TDSZ \\
\hline TDS \\
\hline TDS \\
\hline $\begin{array}{l}\text { Physical serial } \\
\text { map segment }\end{array}$
\end{tabular}

(b)
FIgURE 6: Mapping a zero coefficient. (a) Symbolic serial map segment for a zero-valued coefficient. (b) Corresponding physical serial map segment of a zero-valued coefficient.

a coefficient multiplier for the coefficient 1 and a tap adder slice from which the filter output is obtained.

\subsubsection{Physical serial map}

The physical serial map of Figure $5 \mathrm{c}$ is constructed by representing each core in the symbolic serial map of Figure $5 \mathrm{~b}$ by the number of LEs of FPGA hardware it requires. For example, the Vcc core requires one LE of FPGA hardware, represented by one block in the physical serial map. The two's core requires two LEs of FPGA hardware and is represented by two blocks in the physical serial map of Figure $5 \mathrm{c}$.

\subsubsection{Mapping zero-valued coefficients}

Hardware resources can be saved in the filter architecture of Figure 1 when implementing zero-valued coefficients. A zero-valued coefficient implies the multiplication of the serial input by zero, resulting in a zero product. The coefficient multiplier and tap adder slice can be eliminated and the TDS to the left and right of the zero coefficient are connected with the latency of the tap adder slice included in one of the TDSs. The mapping of a zero coefficient appears in Figure 6.

In Figure 6, an example segment for both symbolic and physical serial maps is presented for a zero-valued coefficient. The symbolic serial map in Figure 6a shows a TDS and a tap delay slice for zero-valued coefficients (TDSZ). The difference between these slices is the length of the delay they implement. The TDSZ is one bit longer because it absorbs the latency of one for the tap adder slice that is removed.

\subsubsection{Mapping duplicate coefficients}

Figure 1 shows the sharing of coefficient multipliers for duplicate coefficients in the transposed filter architecture. Sharing coefficient multipliers for duplicate coefficients leads to significant reductions in hardware resources used to construct symmetrical coefficient FIR filters. Coefficient multiplier sharing is visualized for a set of coefficients $\{1,-1,-1,3\}$ in Figure 5. The coefficient set $\{1,-1,-1,3\}$ has one duplicate coefficient -1 which does not require an exclusive coefficient multiplier. The symbolic serial map of such a coefficient set is shown in Figure 5b. Note that above the sixth core from the bottom of the symbolic serial map in Figure $5 \mathrm{~b}$, a core is mapped to create a coefficient multiplier for the coefficient -1 (a two's core). Below this core, the symbolic serial map of Figure $5 \mathrm{~b}$ has a tap adder slice and TDS pair, followed by another tap adder slice and TDS pair. Both tap adder slices will be connected to the output of the coefficient multiplier for coefficient -1 as shown in the filter architecture of Figure $4 \mathrm{a}$. The physical serial map of Figure $5 \mathrm{c}$ has 23 blocks, which corresponds to 23 LEs of FPGA hardware required to construct the filter. If coefficient multiplier sharing was not used to construct the filter, an additional block would appear in the physical serial map to construct a second multiplier for the duplicate coefficient -1 . The extra block would correspond to an additional two LEs of FPGA hardware required to construct the filter. As the size of the duplicate coefficient increases, hardware savings from sharing coefficient multipliers also increase.

\subsubsection{Mapping fanout buffers}

The transposed filter architecture of Figure 1 might appear to be perfect if it were not for the input fanout problem it presents in implementation. Loading from input fanout reduces the rate that the system clock can operate at, and must be compensated for in situations of excessive fanout. Recall that within an FPGA each additional input connected to an output signal increases the capacitive loading on the output signal driver in addition to the loading already present from the interconnect. The problem of input fanout is less severe in the direct form architecture, where the registers in the delay line serve to insulate the input signal from the effects of fanout.

A bit-serial FIR filter implementation presents its own fanout issue for the requisite control signals. In a filter with many coefficients or very large coefficients, the control signal fanout rises considerably and can be a factor in the overall system performance because of the aforementioned loading problem.

The control signals and input signals are distributed within the FIR filter core through a single layer of flip-flops that buffer these signals against the effects of fanout. The serial data input and the control signal input to the FIR filter core are each connected to a flip-flop. The flip-flop outputs are then connected to the appropriate inputs of the arithmetic operator cores within the FIR filter core. When the number of operator cores connected to the flip-flop outputs exceeds a preset number of allowable connections (the maximum fanout parameter), a new flip-flop is inserted into the design and connected to the appropriate data input or control signal input. In this way, the ratio of signal inputs to outputs can be controlled through the parameterization of RTP 


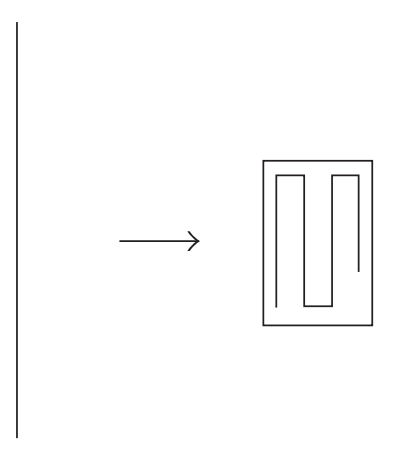

Figure 7: Folding a column of hardware to fit in a rectangular bounding box.

cores [11]. Because of this fanout compensation, the latency of the filter is increased by one time unit.

The TDS core reserves both LEs within a slice because it is implemented with 16-bit SRL16s. See the Xilinx libraries guide online at http://www.xilinx.com/support/ software_manuals.htm. SRL16s are proprietary to Xilinx Virtex devices and require that the slice be placed in a special mode. A slice that is in the special mode cannot implement ordinary four-input lookup tables. As a result, it is sometimes necessary to insert a core of one LE in height into the design prior to the TDS core. The inserted core positions the TDS core for construction within one slice, thereby averting complications in the construction of TDS cores.

If the inserted core is an empty, placeholder core, hardware density and area efficiency are reduced. Inserting a fanout buffer instead of an empty core allows hardware that would otherwise be unused to be purposeful. This is possible because the flip-flops within the slices that are used to buffer the input and control signals are unaffected by the special mode required for implementing SRL16s.

\subsection{Placement: placement director}

Section 4.1 describes how the serial mapper converts a set of coefficients into a column of components. To fit the column into hardware, the physical serial map can be folded to fit inside a rectangular bounding box. A bounding box is the rectangular area reserved by an RTPcore within an FPGA. It can have dimensions of LE, slice, or CLB. The rectangular bounding box can be arbitrarily sized within the confines of the FPGA. The column folding methodology appears in Figure 7; the vertical line represents the physical serial map, the folded line represents the map folded to fit inside a rectangular bounding box.

Figure 5 shows the serial mapping for the coefficient set $\{1,-1,-1,3\}$. If the technique of Figure 7 is applied to the physical serial map of Figure $5 \mathrm{c}$ to fold it into a bounding box that is three CLBs high and two CLBs wide, the bounding box would appear as in Figure 8.

The bottom left corner of the three CLB high and two CLB wide bounding box of Figure 8 corresponds to the top LE of the physical serial map of Figure $5 \mathrm{c}$. The LE, just above

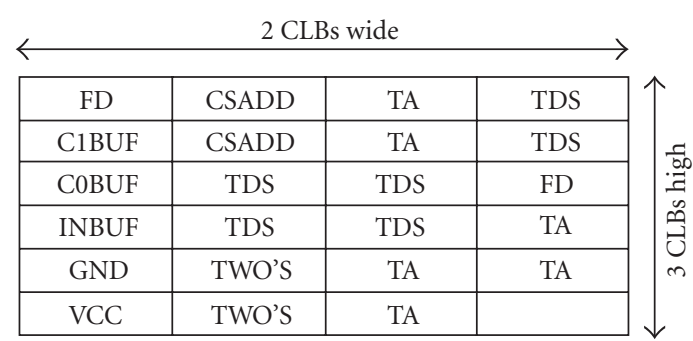

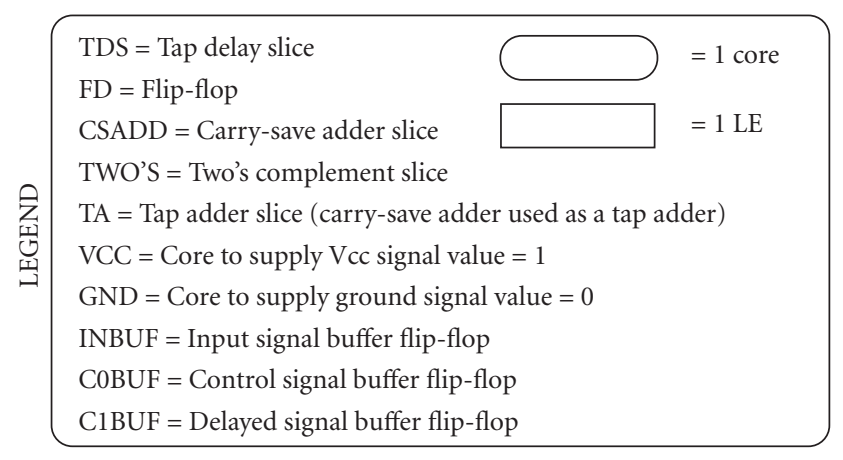

FIgURe 8: The result of folding the physical serial map to fit a bounding box three CLBs high and two CLBs wide.

the bottom left corner LE, corresponds to the next LE in the physical serial map. The first column of the bounding box is filled from the bottom to the top with LEs from the physical serial map until the top is reached. Then placement moves one column to the right and proceeds from the top to the bottom until the bottom is reached. Then placement will move another column to the right and continue until all the cores in the physical serial map are placed in the bounding box.

The placement director is responsible for implementing the aforementioned placement strategy. A column height in CLBs and a starting coordinate corresponding to the bottom left corner of the bounding box must be specified for the placement director to work. The director is then called to generate a coordinate for each core placement based on the size of the core and the current coordinate location.

\subsection{Routing:JRoute}

Routing is the process of assigning wires within the FPGA to create interconnections between the cores placed by the placement director. After the cores are physically placed in a bounding box within the FPGA configuration bitstream by the placement director, the routing process is accomplished using the JRoute tool included with the JBits API. There is no interplay between the placement director and JRoute. For further information, refer to [16].

The placement of the cores within a bounding box in the FPGA will change when the size of the bounding box is changed. This will result in different routing for different bounding box specifications. When distance between two cores that must be connected increases, the timing delay of 
TABLE 2: Hardware cost and PSR results for proposed rapid prototyping design method for Adams' filter (95 taps, passband ripple = $1 \mathrm{~dB}$, passband cutoff $=0.125 \pi \mathrm{rad}$, stopband cutoff $=0.1608 \pi \mathrm{rad}$, and minimum stopband attenuation $=43.22 \mathrm{~dB}$ ).

\begin{tabular}{cc}
\hline Hardware cost (LEs) & PSR $(\mathrm{dB})$ \\
\hline 1144 & 49.9 \\
865 & 48.6 \\
668 & 41.7 \\
\hline
\end{tabular}

the corresponding interconnection also increases. As a result, different bounding box specifications result in different placements that can result in different routing and consequently variations in the timing performance of the core.

\section{PSR AND HARDWARE COST TRADE-OFF}

Table 2 shows the trade-off between the PSR and the hardware cost (the number of LEs required to implement the filter) for Adams' filter [7] (95 taps, passband ripple = $1 \mathrm{~dB}$, passband cutoff $=0.125 \pi \mathrm{rad}$, stopband cutoff $=$ $0.1608 \pi \mathrm{rad}$, minimum stopband attenuation $=43.22 \mathrm{~dB}$ ). Each entry in Table 2 satisfies the frequency response constraints ((2)).

The PSR varies as a direct result of manipulating the value of MaxLE for the proposed method. Tolerating a slight reduction of $1.3 \mathrm{~dB}$ in the PSR results in a significant reduction of the hardware cost by $24 \%$. If the application does not require a high PSR, then the filter requiring 668 LEs can be used. This filter is $42 \%$ smaller than the filter requiring 1144 LEs.

Figures 9 and 10 show the magnitude frequency response of the largest filter, requiring 1144 LEs, and the smallest filter, requiring 668 LEs, using the proposed design method.

\section{FPGA LAYOUT OF A PCLS BIT-SERIAL FIR FILTER CORE}

It is possible to visualize the implementation of a PCLS bitserial FIR filter core in the JBits Boardscope tool [17]. Operational verification of the core is also possible in the Boardscope environment using the virtex device simulator (VirtexDS) [18]. Figure 11 illustrates the packing density of the bit-serial library components as they are placed in a PCLS bit-serial FIR filter core with 95 taps and a PSR of $49.9 \mathrm{~dB}$. The only unused area of the FPGA within the bounding box is the eight LEs at the bottom right corner of the box.

The core pictured in Figure 11 occupies 1071 LEs if fanout buffers are not counted. The bounding box of the core is 18 CLBs wide and 16 CLBs high. The fanout for the pictured core has been limited to a maximum of 25 input nets for any output signal resulting in 73 additional LEs for fanout buffers. The bounding box contains 1152 LEs, including fanout buffers; the filter occupies 1144 LEs (eight LEs are allocated but are unused in this implementation).

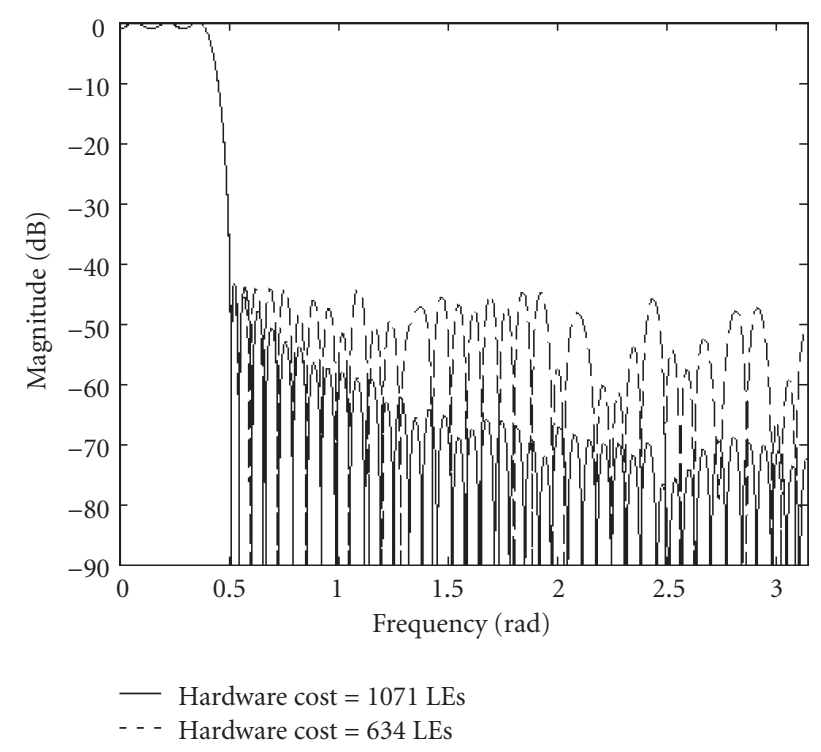

Figure 9: Magnitude frequency response for the filters with the hardware cost of 1144 and 668 LEs for Adams' filter (95 taps, passband ripple $=1 \mathrm{~dB}$, passband cutoff $=0.125 \pi \mathrm{rad}$, stopband cutoff $=0.1608 \pi \mathrm{rad}$, and minimum stopband attenuation $=$ $43.22 \mathrm{~dB})$.

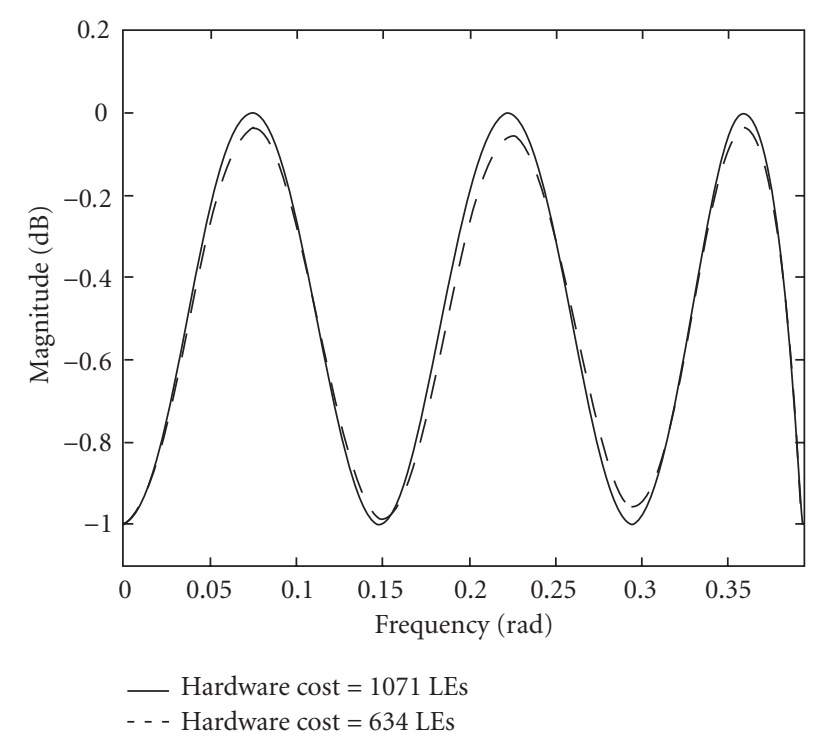

FIGURE 10: Magnitude frequency response of the passband for the filters with the hardware cost of 1144 and 668 LEs for Adams' filter (95 taps, passband ripple $=1 \mathrm{~dB}$, passband cutoff $=0.125 \pi$ $\mathrm{rad}$, stopband cutoff $=0.1608 \pi \mathrm{rad}$, and minimum stopband attenuation $=43.22 \mathrm{~dB}$ ).

Using the method presented in this paper, the 95 tap PCLS bit-serial FIR digital filter can be designed and the bitstream can be created in approximately 4 minutes using a $950 \mathrm{MHz}$ AMD Duron PC. 


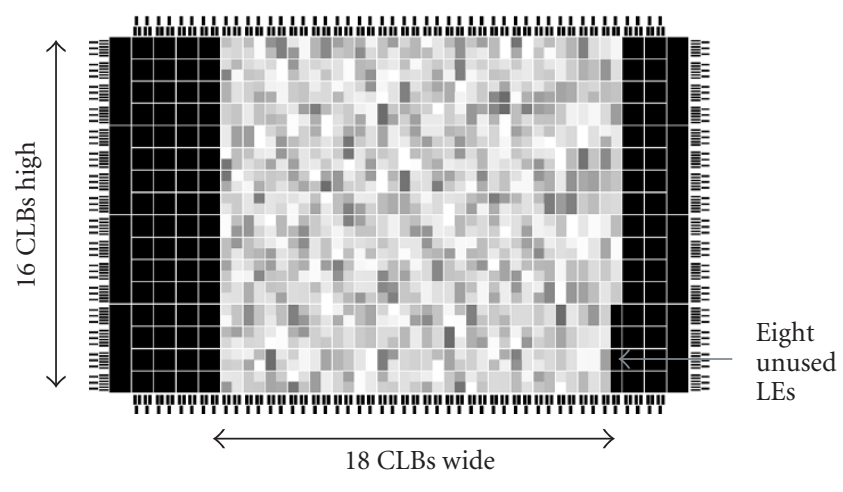

FIGURE 11: Visualization of bit-serial component library subcores as they are placed in a bit-serial FIR filter core with 95 taps and a PSR of $49.9 \mathrm{~dB}$. The device shown is the VirtexDS simulation of the Xilinx Virtex XCV50 part, the smallest Virtex device.

\section{REFERENCES}

[1] A. Antoniou, Digital Filters, Analysis, Design, and Applications, McGraw-Hill, New York, NY, USA, 1993.

[2] R. J. Andraka, "FIR filter fits in an FPGA using a bit serial approach," in Proc. 3rd Annual PLD Conference, Manhasset, NY, USA, March 1993.

[3] S. He and M. Torkelson, "FPGA implementation of FIR filters using pipelined bit-serial canonical signed digit multipliers," in Custom Integrated Circuits Conference (CICC '94), pp. 8184, San Diego, Calif, USA, May 1994.

[4] Y. C. Lim, J. B. Evans, and B. Liu, "An efficient bit-serial FIR filter architecture," Circuits, Systems, and Signal Processing, vol. 14, no. 5, pp. 639-651, 1995.

[5] P. B. James-Roxby, "Designing application-specific cores using JBits: a run-time parameterizable FIR filter," in Reconfigurable Technology: FPGAs and Reconfigurable Processors for Computing and Communications III, vol. 4525 of SPIE Proceedings, pp. 18-26, Denver, Colo, USA, August 2001.

[6] J. W. Adams and J. L. Sullivan, "Peak-constrained least squares optimization," IEEE Trans. Signal Processing, vol. 46, pp. 306 321, February 1998.

[7] J. W. Adams, "FIR digital filters with least-squares stopbands subject to peak-gain constraints," IEEE Trans. Circuits and Systems, vol. 39, no. 4, pp. 376-388, 1991.

[8] T. W. Fox and L. E. Turner, "The design of peak constrained least squares FIR filters with low complexity finite precision coefficients," in Proc. IEEE Int. Symp. Circuits and Systems, vol. 2, pp. 605-608, Sydney, Australia, May 2001.

[9] T. W. Fox and L. E. Turner, "The design of peak constrained least squares FIR filters with low complexity finite precision coefficients," IEEE Transactions on Circuits and Systems II, vol. 49, pp. 151-154, February 2002.

[10] R. I. Hartley and K. K. Parhi, Digit-Serial Computation, Kluwer Academic Publishers, Boston, Mass, USA, 1995.

[11] S. A. Guccione and D. Levi, "Run-Time Parameterizable cores," in Proc. 9th International Workshop on FieldProgrammable Logic and Applications, FPL '99, pp. 215-222, Glasgow, UK, August-September 1999.

[12] S. A. Guccione, D. Levi, and P. Sundararajan, "JBits: Javabased interface for reconfigurable computing," in 2nd Annual Military and Aerospace Applications of Programmable Devices and Technologies (MAPLD '99), The Johns Hopkins University, Laurel, Md, USA, September 1999.

[13] J. B. Ballagh, "An FPGA-based run-time reconfigurable 2-D discrete wavelet transform core," M.S. thesis, Virginia Poly- technic Institute and State University, Blacksburg, Va, USA, June 2001.

[14] J. Valls, M. M. Peiro, T. Sansaloni, and E. Boemo, "Design and FPGA implementation of digit-serial FIR filters," in Proc. 5th IEEE International Conference on Electronics, Circuits and Systems (ICECS '98), vol. 2, pp. 191-194, Lisboa, Portugal, September 1998.

[15] Virtex $^{\mathrm{TM}} 2.5$ V Field Programmable Gate Arrays-Final Product Specification, May 2000, http://www.xilinx.com.

[16] E. Keller, "JRoute: A run-time routing API for FPGA hardware," in Parallel and Distributed Processing, J. Romlin et al., Eds., vol. 1800 of Lecture Notes in Computer Science, pp. 874881, Springer-Verlag, Berlin, May 2000.

[17] D. Levi and S. A. Guccione, "BoardScope: a debug tool for reconfigurable systems," in Configurable Computing Technology and Its Uses in High Performance Computing, DSP and Systems Engineering, Proc. SPIE Photonics East, J. Schewel, Ed., vol. 3526 of SPIE Proceedings, Bellingham, Wash, USA, November 1998.

[18] S. McMillan, B. Blodget, and S. Guccione, "VirtexDS: a device simulator for Virtex," in Reconfigurable Technology: FPGAs for Computing and Applications II, vol. 4212 of SPIE Proceedings, pp. 50-56, Bellingham, Wash, USA, November 2000.

Alex Carreira received a B.S. degree in electrical engineering from the University of Calgary, Canada in 1999. He is presently completing an M.S. degree in electrical engineering at the University of Calgary. His main research interests are digital signal processing with programmable logic devices, configurable and reconfigurable computing, and rapid prototyping of systems for programmable logic devices.

Trevor W. Fox received the B.S. and Ph.D. degrees in electrical engineering from the University of Calgary in 1999 and 2002, respectively. He is presently working for Intelligent Engines in Calgary, Canada. His main research interests include digital filter design, reconfigurable digital signal processing, and rapid prototyping of digital systems.

Laurence E. Turner received the B.S. and Ph.D. degrees in electrical engineering from the University of Calgary in 1974 and 1979, respectively. Since 1979, he has been a faculty member at the University of Calgary where he currently is a Full Professor in the Department of Electrical and Computer Engineering. His research interests include digital filter design, finite precision effects in digital filters, and the development of computer-aided design tools for digital system design.
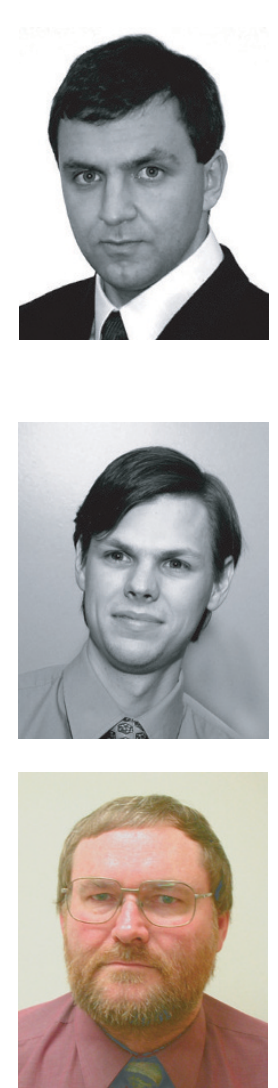